\title{
Basophils balance healing after myocardial infarction via IL-4/IL-13
}

\author{
Florian Sicklinger, ${ }^{1,2}$ Ingmar Sören Meyer, ${ }^{1,2}$ Xue Li, ${ }^{1,2}$ Daniel Radtke, ${ }^{3}$ Severin Dicks, ${ }^{4,5}$ Moritz P. Kornadt, ${ }^{1,2}$ Christina Mertens, ${ }^{6}$ \\ Julia K. Meier, ${ }^{7}$ Kory J. Lavine, ${ }^{8}$ Yunhang Zhang, ${ }^{1,2}$ Tim Christian Kuhn, ${ }^{1,2}$ Tobias Terzer, ${ }^{9}$ Jyoti Patel, ${ }^{1,2}$ Melanie Boerries, ${ }^{4,10}$ \\ Gabriele Schramm, ${ }^{11}$ Norbert Frey, ${ }^{1,2}$ Hugo A. Katus, ${ }^{1,2}$ David Voehringer, ${ }^{3}$ and Florian Leuschner ${ }^{1,2}$

\begin{abstract}
'Department of Cardiology, University Hospital Heidelberg, Heidelberg, Germany. ${ }^{2}$ Cerman Centre for Cardiovascular Research (DZHK), Partner Site Heidelberg, Heidelberg, Germany. ${ }^{3}$ Department of Infection Biology, University Hospital Erlangen and Friedrich Alexander University Erlangen-Nuremberg (FAU), Erlangen, Germany. ${ }^{4}$ Institute of Medical Bioinformatics and Systems Medicine, Medical Center and Faculty of Medicine, University of Freiburg, Freiburg, Germany. ${ }^{5}$ Faculty of Biology, Albert Ludwig University, Freiburg, Cermany. ${ }^{6}$ Department of Pediatric Hematology, Oncology, and Immunology, University of Heidelberg, Heidelberg, Germany. ${ }^{7}$ Institute of Biochemistry I, Goethe-University Frankfurt, Faculty of Medicine, Frankfurt am Main, Germany. ${ }^{8}$ Department of Medicine, Washington University, St. Louis, Missouri, USA. ${ }^{9}$ Division of Biostatistics, German Cancer Research Center (DKFZ), Heidelberg, Germany. ${ }^{10}$ Cerman Cancer Consortium (DKTK) Partner Site Freiburg, DKFZ, Freiburg, Germany. "Experimental
\end{abstract} \\ Pneumology, Research Center Borstel, Airway Research Center North, Member of the German Center for Lung Research (DZL), Borstel, Cermany.
}

\begin{abstract}
The inflammatory response after myocardial infarction (MI) is a precisely regulated process that greatly affects subsequent remodeling. Here, we show that basophil granulocytes infiltrated infarcted murine hearts, with a peak occurring between days 3 and 7. Antibody-mediated and genetic depletion of basophils deteriorated cardiac function and resulted in enhanced scar thinning after MI. Mechanistically, we found that basophil depletion was associated with a shift from reparative Ly $6 C^{10}$ macrophages toward increased numbers of inflammatory Ly $6 \mathrm{C}^{\text {hi }}$ monocytes in the infarcted myocardium. Restoration of basophils in basophil-deficient mice by adoptive transfer reversed this proinflammatory phenotype. Cellular alterations in the absence of basophils were accompanied by lower cardiac levels of IL-4 and IL-13, two major cytokines secreted by basophils. Mice with basophil-specific IL-4/IL-13 deficiency exhibited a similarly altered myeloid response with an increased fraction of Ly6C $\mathrm{Ch}^{\mathrm{i}}$ monocytes and aggravated cardiac function after MI. In contrast, IL-4 induction in basophils via administration of the glycoprotein IPSE/ $\alpha-1$ led to improved post-MI healing. These results in mice were corroborated by the finding that initially low counts of blood basophils in patients with acute MI were associated with a worse cardiac outcome after 1 year, characterized by a larger scar size. In conclusion, we show that basophils promoted tissue repair after MI by increasing cardiac IL-4 and IL-13 levels.
\end{abstract}

\section{Introduction}

Inflammation is an essential mechanism for restoring tissue integrity after myocardial infarction (MI). After restriction of blood supply, the inflammatory response is characterized by a multistep cascade at both the cellular and molecular levels (1). Following an early accumulation of neutrophils, peripheral monocytes are attracted by various stimuli, enter the injured heart, and further amplify the inflammatory response $(2,3)$. After several days, the proinflammatory phase is superseded by a reparative phase in which monocyte-derived macrophages constitute the predominant immune cell type in the heart (4). These latter cells have a rather antiinflammatory program and provide a palette of mediators that promote angiogenesis and collagen deposition, thereby contributing to the formation of a solid scar (5). Recent studies have shown that other leukocyte populations, such as DCs, mast cells, B cells, T cells, NK cells, and Tregs, also play important roles in MI healing (6-10). Despite their quantitative inferiority com-

Conflict of interest: The authors have declared that no conflict of interest exists. Copyright: @ 2021, American Society for Clinical Investigation.

Submitted: January 27, 2020; Accepted: May 6, 2021; Published: July 1, 2021.

Reference information: / Clin Invest. 2021;131(13):e136778.

https://doi.org/10.1172/JCl136778. pared with monocytes/macrophages at the site of inflammation, these cells either have direct potent effector functions or indirectly coordinate the response of other immune cells. However, a more comprehensive characterization of cellular heterogeneity and interaction in the post-MI inflammatory network remains an important objective. A better understanding of how immune cells cooperate to restore tissue function and how the reaction of individual cell types is integrated to yield a coordinated tissue-scale response following injury has important implications - not only for the identification of new cellular and molecular targets, but also for the successful implementation of promising immunomodulatory therapies in patients with MI.

Basophils are blood-borne immune cells and were first described by Paul Ehrlich more than 130 years ago (11). The role of these cells in health and disease has long been an enigma, but research over the past 2 decades has shed more light on basophil biology (12). Basophils are conserved in all vertebrates, have a relatively short lifespan of 2-3 days, and can be rapidly mobilized in inflammatory conditions (13). They express the high-affinity IgE receptor FceRI and can produce a set of defined effector molecules, including proteolytic enzymes, bioactive lipids (prostaglandins and leukotrienes), histamine, TNF- $\alpha$, IL-6, IL-4, and IL-13. Basophils are best known to be critical effector cells in allergic 
A

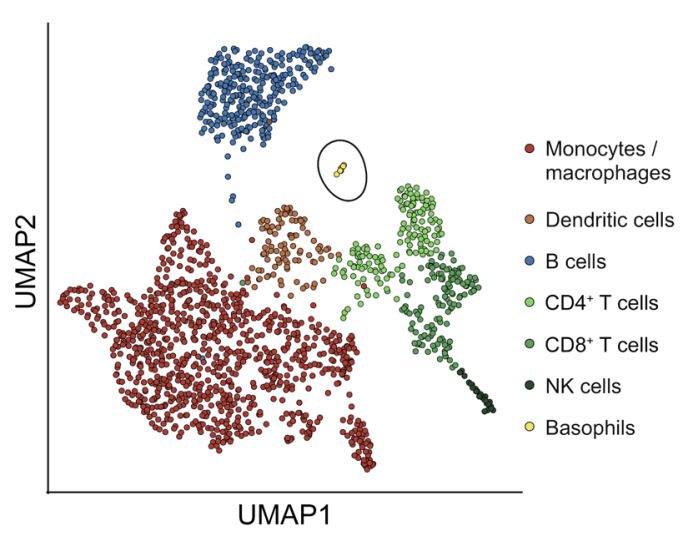

C

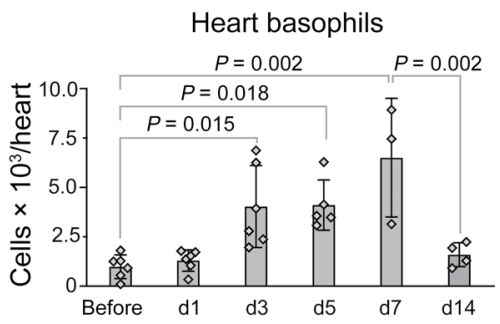

B Before
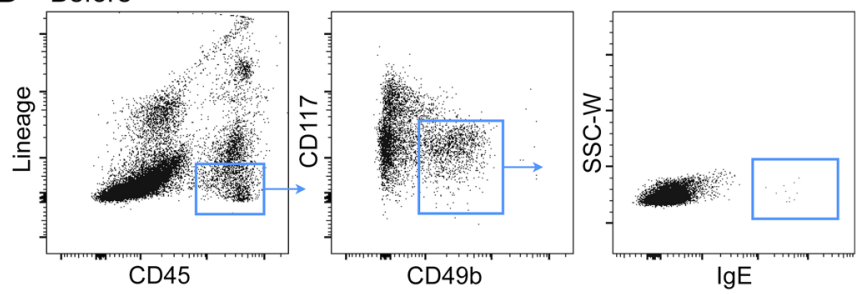

Day 3 after MI
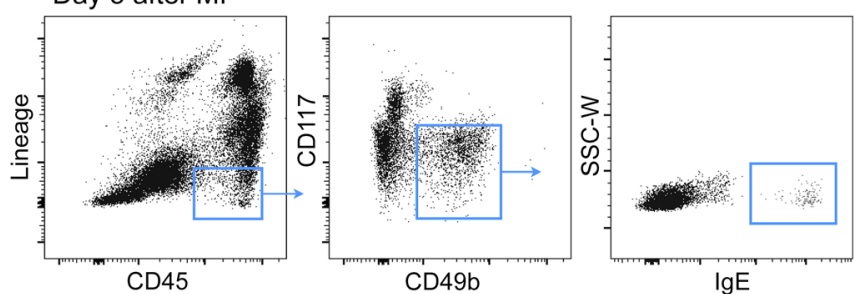

D

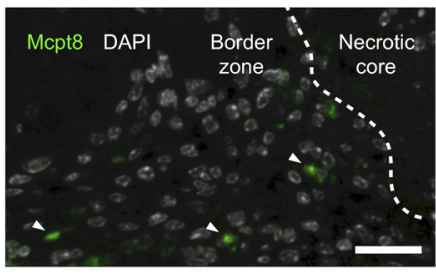

Figure 1. Basophil granulocytes infiltrate the heart after permanent LAD ligation in mice. (A) UMAP embedment of single-cell RNA-Seq data for cells isolated from the infarct region of a WT mouse heart 7 days after MI. (B) Representative images of flow cytometric analysis of heart tissue before and

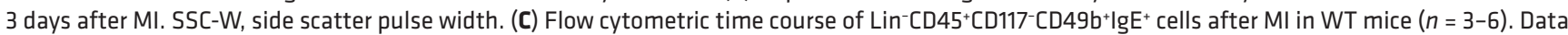
show the mean \pm SD. $P$ values were determined by 1-way ANOVA with Sidak's multiple-comparison post hoc test. (D) Immunohistochemical staining of Mcpt8 ${ }^{+}$cells (green, arrowhead) in the infarct region 7 days after MI. Nuclei are depicted in white (DAPI). Scale bar: $20 \mu \mathrm{m}$. Data show the mean \pm SD. $P$ value was determined by 2 -tailed Student's $t$ test $(n=3)$.

reactions and protective immunity against parasites $(14,15)$. In addition, recent studies suggest that basophils are highly interactive cells and may provide unique functions unmet by other hematopoietic cells, particularly in immune imprinting, host response to bacteria, autoimmune disease, and allograft fibrosis (16-20). Their role in cardiovascular diseases, however, has not been mechanistically explored to date.

In this study, we found that basophil counts increased in the infarcted myocardium within a few days after MI. With the use of antibody depletion and mice genetically deficient in basophils, we show that basophils were essential for competent MI healing, mainly by altering local monocyte/macrophage responses. Further, experiments in mice with basophil-specific IL-4/IL-13 deficiency indicated that these cytokines were essential mediators for basophil-dependent macrophage polarization after MI. These data provide insights into how basophils coordinate innate immune responses during sterile inflammation and wound healing after MI.

\section{Results}

Basophil granulocytes infiltrate the heart after MI in mice. To examine the cellular composition of different immune cell types in MI healing, we performed an analysis of a single-cell RNA-Seq data set of cardiac leukocytes during the reparative phase after murine
MI (21). Consistent with prior studies, the immune compartment in the infarcted myocardium consisted mainly of monocytes, macrophages, DCs, B cells, T cells, and NK cells (Figure 1A). However, unbiased clustering of all leukocytes based on unique patterns of gene expression also indicated the presence of a basophil cluster marked by the expression of $M c p t 8$, which encodes mast cell protease 8 and other basophil markers (Figure 1A, Supplemental Figure 1, A-C, and Supplemental Table 1; supplemental material available online with this article; https://doi.org/10.1172/JCI136778DS1). In order to corroborate whether the latter population in fact accumulates after myocardial ischemia, we studied infarcted hearts by flow cytometry. Basophils were identified as $\mathrm{CD} 45^{\text {int } / \text { hi }}$ Lin ${ }^{-} D 117^{-} \mathrm{CD}^{2} 9 \mathrm{~b}^{+} \mathrm{IgE} \mathrm{E}^{+}$cells (Figure 1B) (14). After permanent ligation of the left anterior descending artery (LAD), enumeration based on flow cytometry revealed an increase in basophil counts in infarcted hearts, with a peak occurring between days 3 and 7 and a return to baseline on day 14 (Figure 1C). Immunohistochemical staining confirmed the presence of $\mathrm{Mcpt}^{+}$cells in infarcted hearts (Figure 1D). Basophils express a variety of cytokine receptors and can therefore respond to numerous extrinsic signals that regulate their recruitment, degranulation, and cytokine production (22). Among the main mediators known for homing of basophils to inflamed tissue (IL-3, thymic stromal lymphopoietin, IL-33), we could identify IL-33 as a cytokine markedly upregulated in the 
A
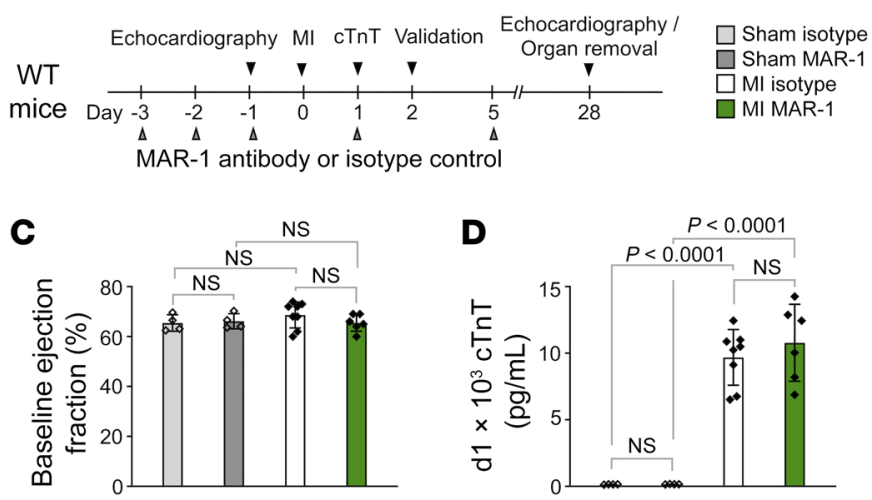

E
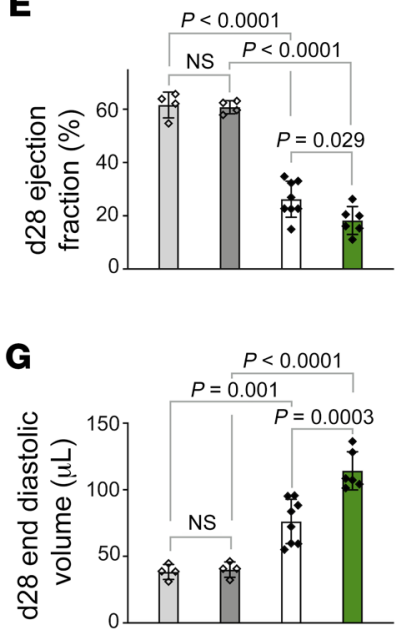

$\mathbf{F}$

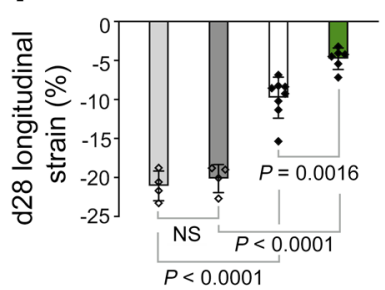

B

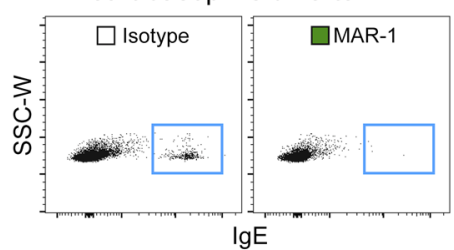

I
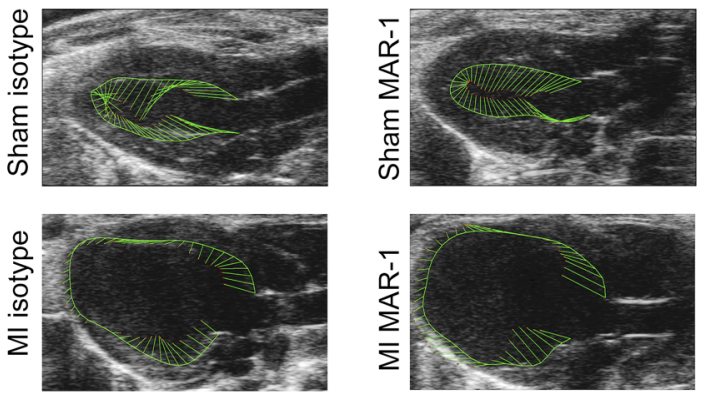

J

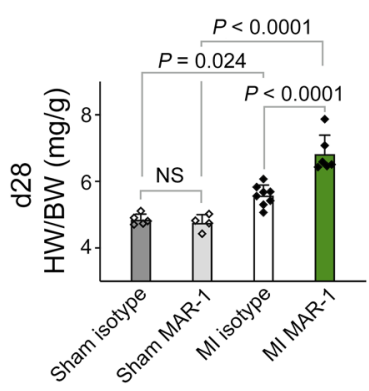

K

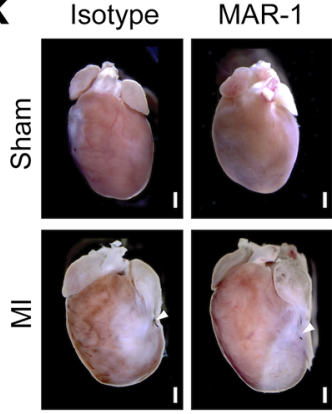

Figure 2. Basophil depletion by antibody injection worsens cardiac function after acute $\mathrm{MI}$ in mice. (A) Timeline of basophil depletion experiments. (B) Frequencies of basophils from of hearts of IgG-injected control and anti-FcERI-injected animals were assessed by flow cytometry 2 days (d2) after MI $(n=4)$. Data show the mean \pm SD. $P$ value was determined by 2-tailed Student's $t$ test. (C) Echocardiographic evaluation of baseline EF in IgG- and MAR-1treated mice. (D) Plasma levels of $\mathrm{CTnT}$ in IgG- and MAR-1-treated mice were measured 24 hours after LAD ligation or sham intervention. $P$ values were determined by 2-way ANOVA followed by Tukey's multiple-comparison test. (E-H) Echocardiographic results for IgG-treated and MAR-1-treated mice 4 weeks after MI or sham surgery. $P$ values were determined by 2-way ANOVA followed by Tukey's multiple-comparison test. (I) Representative echocardiographic images 4 weeks after MI. Vectors display the direction and magnitude of myocardial contraction at midsystole. (J) Quantification of heart weight to body weight ratio (HW/BW) determined 4 weeks after MI $(n=4-8)$. Data show the mean \pm SD. $P$ values were determined by 2 -way ANOVA followed by Tukey's multiple-comparison test. (K) Representative hearts from IgG- and MAR-1-treated mice 4 weeks after MI or sham intervention. Arrowheads indicate the site of ligation. Scale bars: $500 \mu \mathrm{m}$.

infarcted heart 2 days after MI with the correspondent receptor Il1rl1 expressed in the basophil cluster (Supplemental Figure 1, D and E). Furthermore, Ccl-5, IL-18, and IgE were significantly increased and may be additional stimuli for basophil recruitment and activation after MI (Supplemental Figure 1, F-K).

Antibody-mediated basophil depletion worsens cardiac function after acute MI. To clarify whether basophil accumulation into the infarcted myocardium was a mere bystander effect or had functional consequences, we depleted basophils by injecting the FceRI-specific antibody MAR-1 (Figure 2A). As expected, antiFceRI treatment almost completely abolished the number of basophils in heart, peripheral blood, and spleen under basal conditions (Supplemental Figure 2A) and 2 days after permanent LAD ligation (Figure 2B and Supplemental Figure 2, B-D). Mast cells and a subset of DCs also express the FceRI, however, we detected no significant differences in the number of cardiac mast cells and
DCs between anti-FceRI-treated mice and IgG control-treated mice (Supplemental Figure 2, E-I). Baseline left ventricular (LV) ejection fraction (EF) before surgery and initial infarct sizes on day 1 as measured by cardiac troponin T (cTnT) levels in plasma samples and 2,3,5-triphenyltetrazolium chloride (TTC) staining were not different between the control and basophil-depleted mice (Figure 2, C and D, and Supplemental Figure 3A). Assessment of cardiac function 4 weeks after permanent LAD ligation confirmed reduced cardiac function and enlarged LV geometry accompanied by increased heart weight to body weight ratios in infarcted mice compared with animals after sham intervention. Basophil depletion, however, led to larger end-diastolic and end-systolic volumes and a significant reduction of LV EF compared with isotype control-treated animals. In support of the observed reduced global contractility, we found a significantly greater decrease in global longitudinal strain in infarcted hearts of MAR-1-treated mice 
A

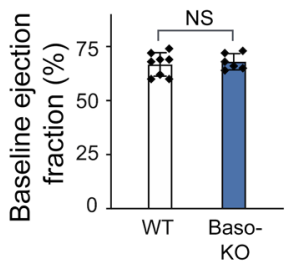

D

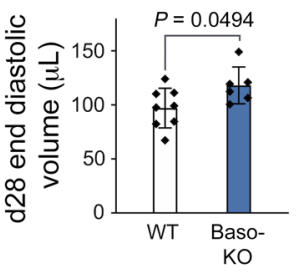

$\mathbf{G}$

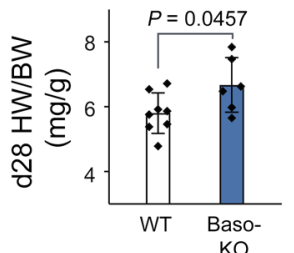

B

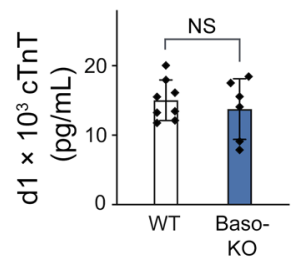

E

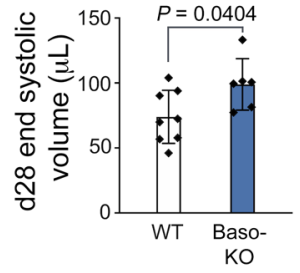

H

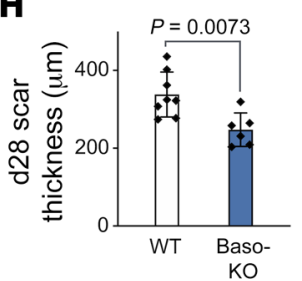

C
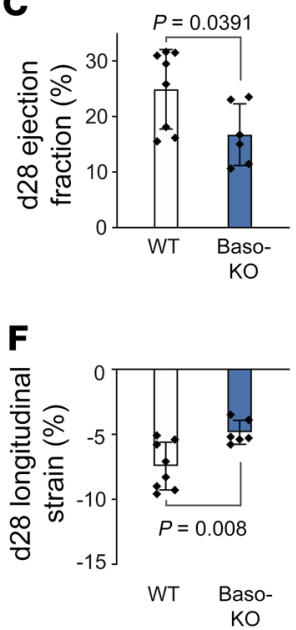

I

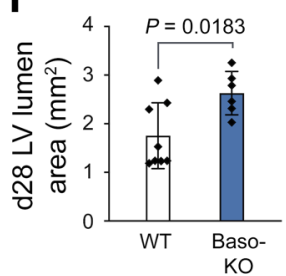

J

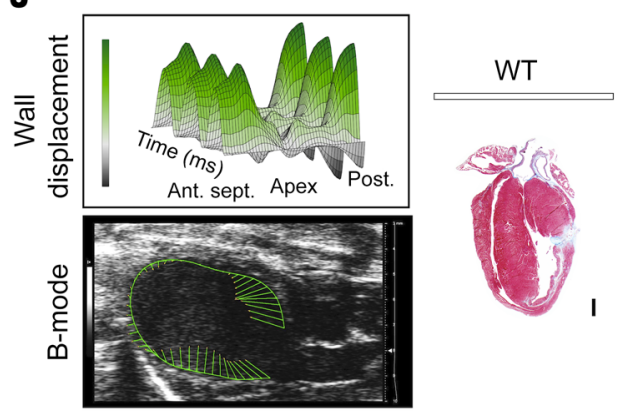

$\mathbf{K}$

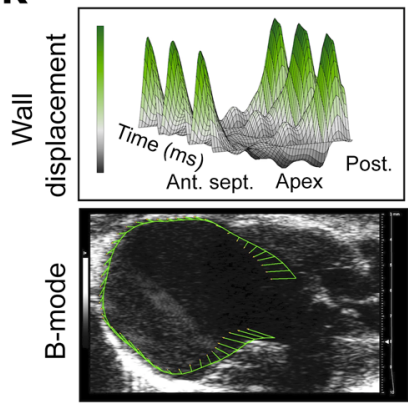

\section{BasoKO}

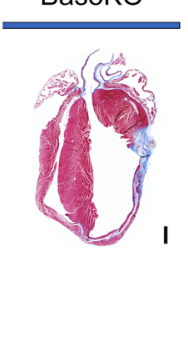

Figure 3. Baso-KO mice show deteriorated healing after MI. (A) Echocardiographic evaluation of baseline EF in WT and Baso-KO mice. (B) Plasma cTnT values in WT and Baso-KO mice measured 24 hours after LAD ligation. (C-F) Echocardiographic results for Baso-KO and WT mice 4 weeks after MI. $P$ values were determined by 2-tailed Student's $t$ test. (G) Quantification of heart weight to body weight ratio 4 weeks after MI. $P$ value was determined by 2-tailed Student's $t$ test. (H and I) Scar thickness and LV lumen area were quantified by histological evaluation 4 weeks after MI ( $n=6$-8). Data show the mean \pm SD. $P$ values were determined by 2-tailed Student's $t$ test. (J and $\mathbf{K}$ ) Representative echocardiographic and histological images of WT and Baso-KO mice 4 weeks after MI. 3D plots show radial displacement over 3 cardiac cycles. Histological sections were stained with Masson's trichrome to detect fibrosis. Scale bars: $500 \mu \mathrm{m}$. Ant. sept., anterior septal; Post., posterior.

compared with the hearts of control-treated mice (Figure 2, E-I, and Supplemental Figure 3B). Additionally, MAR-1-treated mice showed increased heart weight to body weight ratios (Figure 2, J and $\mathrm{K}$ ) and had significantly reduced scar thickness and increased LV lumen area (Supplemental Figure 3, C-E).

Mice with genetic basophil ablation show deteriorated healing after acute MI. Any antibody treatment may have limitations that influence healing processes, such as activation of the complement system, clearance of opsonized apoptotic cells, or phenotypical changes in cells expressing the antibody target (23). To overcome these limitations, we studied Mcpt8-Cre-transgenic (Baso-KO) mice that are constitutively deficient in basophils (14). Under basal conditions Baso-KO mice showed no cardiac-specific phenotype, as evaluated by echocardiography (Figure 3A). Upon LAD ligation, we detected no differences in initial infarct size as measured by cTnT levels in plasma samples 24 hours after infarction compared with WT mice (Figure 3B). However, 28 days after infarction, Baso-KO mice had developed severe cardiac dysfunction marked by significantly reduced LV EF, increased end-diastolic and end-systolic LV volumes, decreased global longitudinal strain, and significantly increased heart weight to body weight ratios as compared with their WT littermates (Figure 3, C-G). Histopathological evaluation, moreover, revealed significantly more LV chamber dilation and exaggerated scar thinning in Baso-KO mice compared with WT controls (Figure 3, H-K).
Genetic basophil ablation alters myeloid cell responses following MI. To investigate the potential mechanism by which basophils contribute to myocardial healing, we studied the cellular inflammatory response in infarcted hearts in the presence and absence of basophils. In order to rule out potential off-target effects of basophil depletion, we additionally established a restoration approach and transferred CD49b+ basophil-enriched splenocytes into BasoKO mice after MI in 1 group (Supplemental Figure 4, A-C). Flow cytometric analysis of murine hearts on day 4 after MI showed unaltered numbers of total CD $45^{+}$leukocytes and $\mathrm{CD} 11 \mathrm{~b}^{+}$myeloid cells in all groups (Figure 4, A-C). However, subpopulation gating revealed a critically altered monocyte/macrophage response, with a significantly elevated presence of Ly6 $\mathrm{C}^{\text {hi }}$ inflammatory monocytes in the absence of basophils. Transfer of basophil-enriched cells into Baso-KO mice resulted in a reversal of the inflammatory phenotype observed in the absence of basophils (Figure 4, D and E). Monocyte levels in blood and bone marrow (BM) as well as vascular adhesion molecules (Vcam-1) in the heart were unchanged, indicating a local and heart-specific response independent of systemic monocytopoiesis (Figure 4, F and G, and Supplemental Figure 5A). Furthermore, Baso-KO mice showed a diminished ratio of antiinflammatory $\mathrm{F} 4 / 80^{+} \mathrm{CD}_{206}{ }^{+}$macrophages among cardiac $\mathrm{F} 4 / 80^{+}$macrophages compared with control animals (Figure $4, \mathrm{H}$ and I). The findings were corroborated by significant changes in the transcriptional profile of hearts from basophil-depleted mice characterized by upregu- 
A
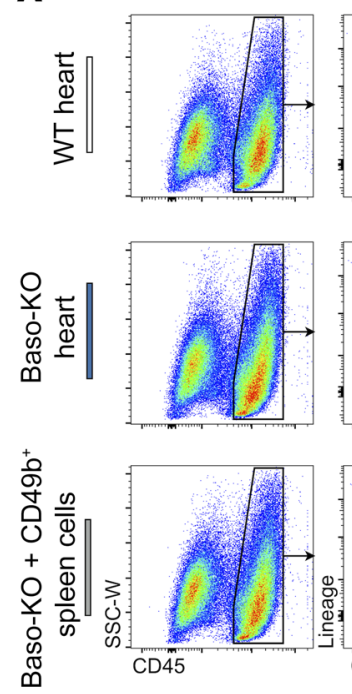

$\mathbf{E}$

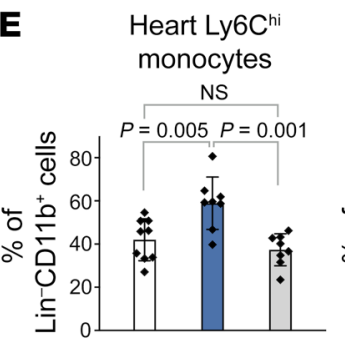

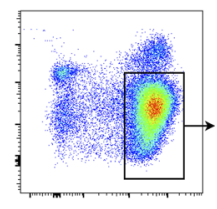
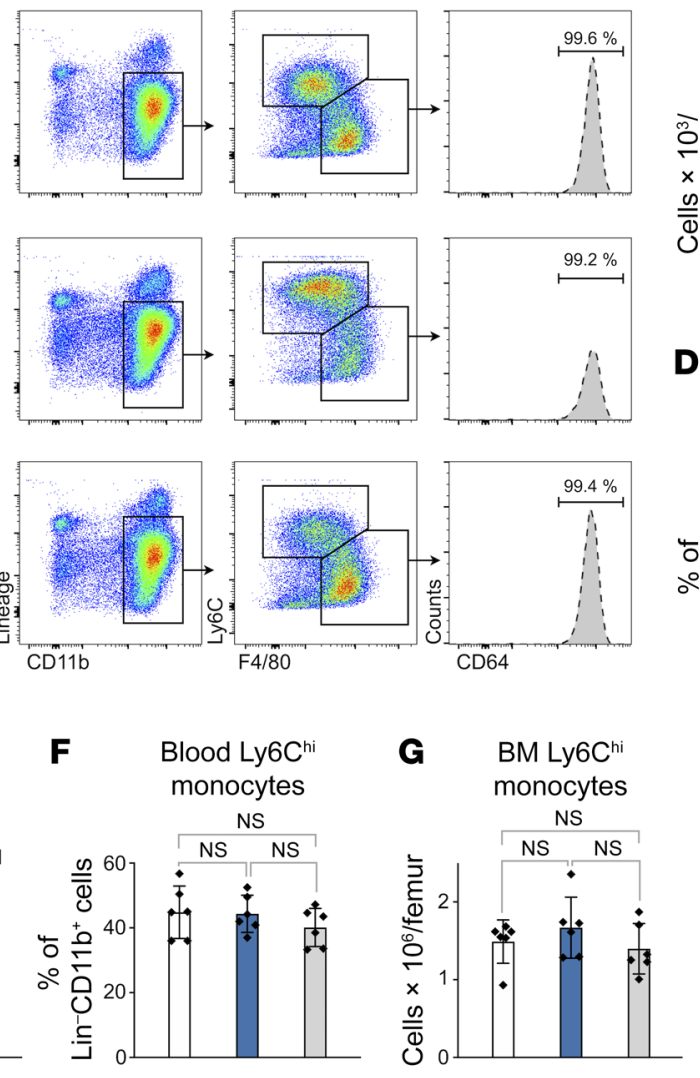

B

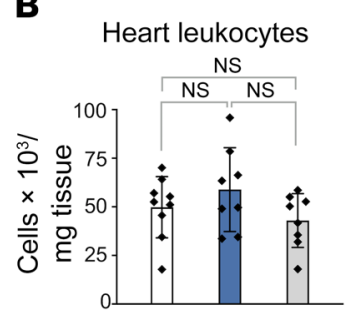

C

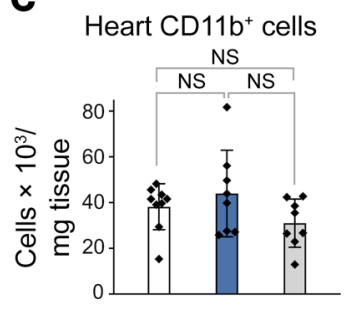

D $\quad \begin{aligned} & \text { Heart Ly6C } \\ & \text { macrophages }\end{aligned}$
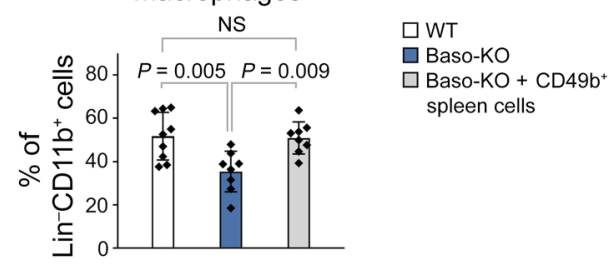
H Gated on $\operatorname{Lin}^{-} \mathrm{CD} 11 \mathrm{~b}^{+} \mathrm{F} 4 / 80^{+}$
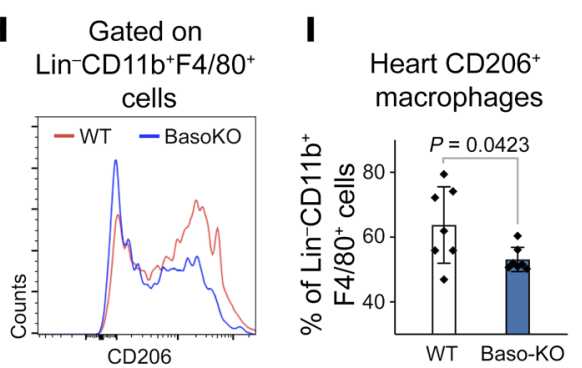

Figure 4. Genetic basophil depletion affects cardiac monocytes and macrophages. (A) Representative flow cytometric plots of infarct tissue 4 days after MI, following transfer of basophil-enriched cells (bottom) gated on monocytes/macrophages. Heart cells were from WT and Baso-KO mice, and splenic cells were from WT mice. (B and C) Quantification of total numbers of $C D 45^{+}$cells or CD45+CD11 b cells per milligram of heart tissue. (D and E) Percentage

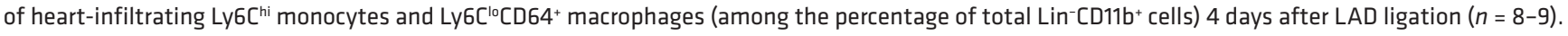
Data indicate the mean \pm SD. $P$ values were determined by 1-way ANOVA followed by Tukey's multiple-comparison test. (F and $\mathbf{G})$ Blood and BM monocyte levels for the indicated groups 4 days after $\mathrm{MI}(n=6)$. (H) Representative histogram depicting CD206+ cells among Lin-CD11 $\mathrm{b}^{+} \mathrm{F} 4 / 80^{+}$macrophages 4 days after MI. (I) Proportion of $\mathrm{CD}_{206^{+}}$cells among Lin-CD11 $\mathrm{b}^{+} \mathrm{F} 4 / 80^{+}$macrophages in the heart 4 days after MI $(n=7)$. Data show the mean \pm SD. $P$ value was determined by 2-tailed Student's $t$ test.

lation of M1 markers (IL-12 $\beta$, IFN- $\gamma$ ) and downregulation of the M2 signature (arginase-1) (Supplemental Figure 5, B-D).

Basophils affect IL-4/IL-13 cytokine production in the injured heart after MI. To evaluate how basophils modulate monocytes/ macrophages, we next assessed the expression of different cytokines and chemokines 3 days after MI. Among all tested cytokines that are known to be secreted by basophils (TNF- $\alpha$, IL-6, IL-4, IL-13), only IL-4 was found to be significantly reduced in the heart of basophil-deficient mice 3 days after MI (Figure 5A). In addition, in the absence of basophils, we detected a significant reduction in the expression of histidine decarboxylase (Hdc), which catalyzes the decarboxylation of histidine to form histamine (Supplemental Figure 5, E and F). To further evaluate these results, we used BasoKO and WT mice on a 4 get background, in which all $\mathrm{IL}^{-} 4^{+}$cells express GFP. Similar to the findings described above, on the mRNA level, the number of $\mathrm{GFP}^{+}$cells after MI was markedly reduced in the hearts of Baso-KO 4get mice compared with control mice (Figure 5, B and C). Gene expression analysis of single-cell RNA-Seq data, moreover, confirmed that IL-4 and IL-13 were detectable mainly in the basophil cluster, whereas genes encoding the cor- respondent cytokine receptors were predominantly expressed in the monocyte/macrophage, DC, and $\mathrm{CD} 4^{+} \mathrm{T}$ cell clusters (Figure 5D). Finally, multiplex immunohistochemistry corroborated IL-4expressing $\mathrm{Mcpt}^{+}$cells in close proximity to $\mathrm{CD} 163^{+}$macrophages (Supplemental Figure 5G). These findings suggest that basophils crucially affect cardiac IL-4 levels after MI, either by direct production or indirectly through other local cytokine-producing cells.

Basophil-specific IL-4/IL-13 deficiency affects the cardiac immune response and aggravates cardiac dysfunction after MI. To directly assess the function of IL-4 and IL-13 derived from basophils, we exploited a previously established approach that allows the in vivo assessment of basophil-specific IL-4/IL-13 deletion (Supplemental Figure 6, A-J) (24). Following permanent LAD ligation, mice with basophils incapable of producing IL-4 or IL-13 showed significantly higher levels of inflammatory Ly $6 \mathrm{C}^{\text {hi }}$ monocytes within the myeloid cell compartment in the infarcted heart, suggesting that the effect of basophils on monocytes/macrophages is mainly driven by basophil-derived IL-4 and IL-13 (Figure 6, A-C). The overall numbers of recruited $\mathrm{CD} 45^{+}$leukocytes and total CD11 $\mathrm{b}^{+}$ myeloid cells remained unaltered (Supplemental Figure 6, K-M). 
A
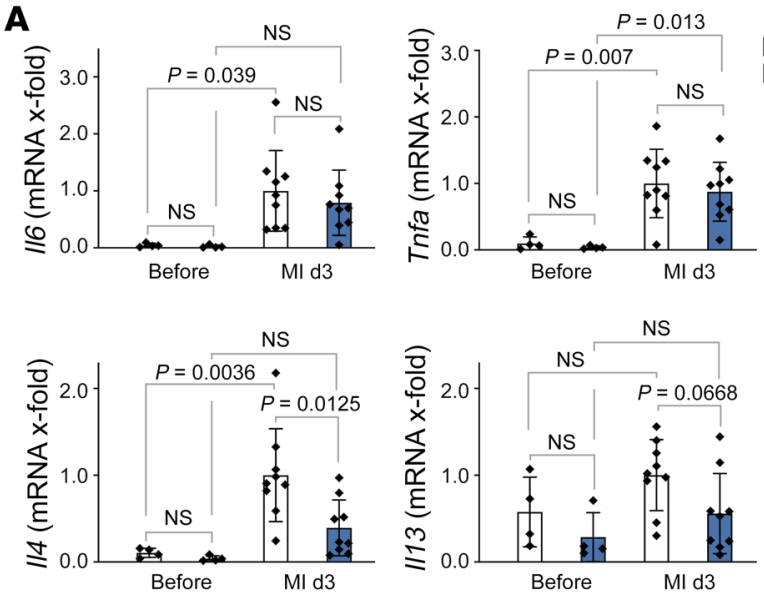

\section{B}

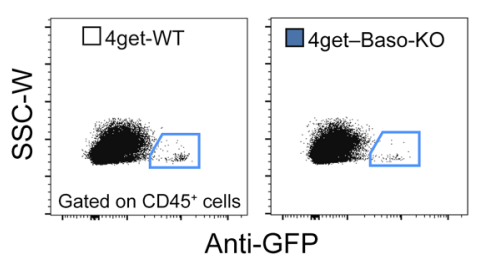

C

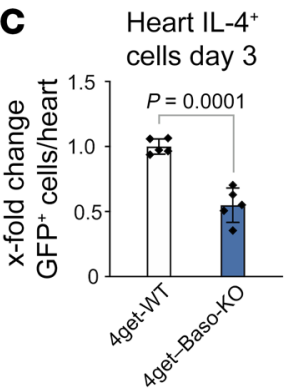

D $\square$ WT
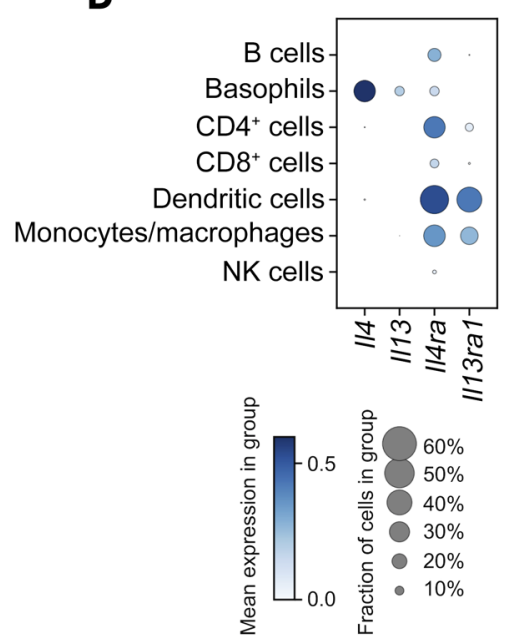

Figure 5. Basophils contribute to IL-4 and IL-13 production in the injured heart after MI. (A) mRNA expression of $/ / 6$, Tnfa, II4, and I/13 in the infarct region 3 days after MI in WT and Baso-KO mice $(n=8-9)$. mRNA levels are expressed as $\mathrm{x}$-fold relative to the WT MI group. Data show the mean \pm SD. $P$ values were determined by 2-way ANOVA followed by Sidak's multiple-comparison test. (B) Representative flow cytometric plots of cells from the infarct region 3 days after MI in WT 4get and Baso-KO 4get mice. (C) Quantification of GFP+ cells in the infarct region 3 days after MI in control WT 4get and Baso-KO 4get mice $(n=5)$. Data show the mean \pm SD. $P$ value was determined by 2-tailed Student's $t$ test. (D) Gene expression dot plot based on single-cell RNA-Seq analysis of mouse cardiac leukocytes 3-7 days after MI. Mean expression is depicted by color intensity, whereas the dot size represents the fraction of cells expressing the indicated gene.

Furthermore, basophil-specific IL-4/IL-13 deficiency exacerbated cardiac dysfunction and resulted in a more pronounced infarct expansion and thinner infarct thickness compared with control mice 28 days after MI (Figure 6, D-K).

$I L-4$ induction in basophils by IPSE/ $\alpha-1$ improves heart function after MI. Given the finding that the absence of basophils impeded the post-MI healing process, we hypothesized that activation of basophils and their production of IL-4/IL-13 may enhance myocardial healing and improve heart function following cardiac injury. Helminth parasites are known to be potent natural inducers of Th2 cytokines, and it was previously shown that IPSE/ $\alpha-1$, a major secretory product from Schistosoma mansoni eggs, can trigger basophils to release IL-4 and IL-13 $(25,26)$. As expected, in vitro stimulation of human basophils purified from healthy donors with IPSE/ $\alpha-1$ with or without IL-33 supplementation led to a robust release of IL-4 and IL-13 (Supplemental Figure 7, A and B). Moreover, supernatant from IPSE/ $\alpha$-1-treated basophils significantly increased the expression of CD206 and CD209 on monocytes compared with supernatant from control-treated basophils (Supplemental Figure 7, C and D). In vivo, post-MI administration of IPSE/ $\alpha-1$ resulted in significantly increased levels of cardiac IL-4 compared with PBS-treated controls (Figure 7, A and B). Further, IPSE/ $\alpha$-1-treated animals showed significantly improved cardiac function and cardiac remodeling 28 days after MI compared with control-treated animals, despite equal initial infarct sizes. These positive effects were lost upon additional administration of antiFceRI antibody, indicating a basophil-dependent mechanism of IPSE/ $\alpha-1$ (Figure 7, C-G, and Supplemental Figure 7, E and F).

Low basophil counts are associated with poor outcomes for patients with acute MI. Finally, we addressed the relevance of these findings for patients with MI. We performed flow cytometric staining of human basophils and prospectively performed analyses of blood from patients presenting with acute MI $(n=11)$ and controls with nonischemic cardiovascular disease $(n=9)$ (Figure $8 \mathrm{~A}$ and Supplemental Table 2). Initial blood basophil counts of patients with $\mathrm{MI}(\mathrm{O} \mathrm{h})$ were in the same range as those of control patients. Interestingly, however, we found that basophil counts gradually increased in subsequent days, reaching a maximum 96 hours after MI (Figure 8B). Furthermore, we detected significantly higher expression of CD63, a human basophil activation marker, on circulating basophils 48 hours after MI compared with control basophils, suggesting functional differences as well (Figure 8C). To explore the potential contribution of basophils to the outcome of human MI healing, we prospectively assessed the association of blood basophil counts during acute MI healing and clinical outcomes in an additional cohort of 82 patients. The patients' base- 
A

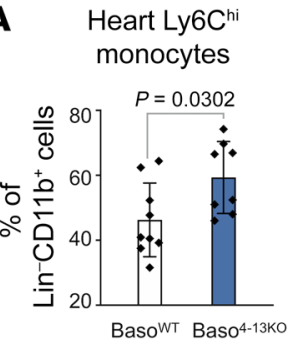

D

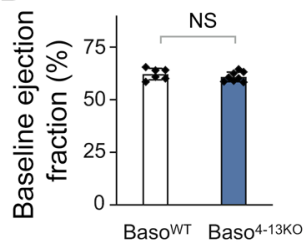

$\mathbf{F}$

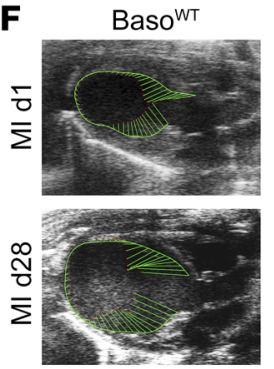

I

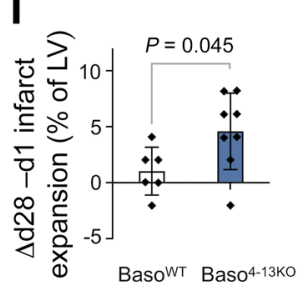

B Heart Ly6C ${ }^{\circ}$

macrophages $P=0.0251$

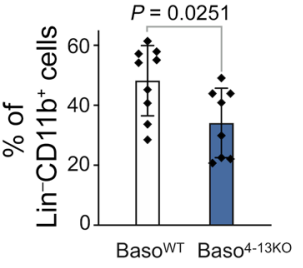

E

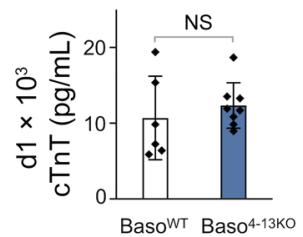

C
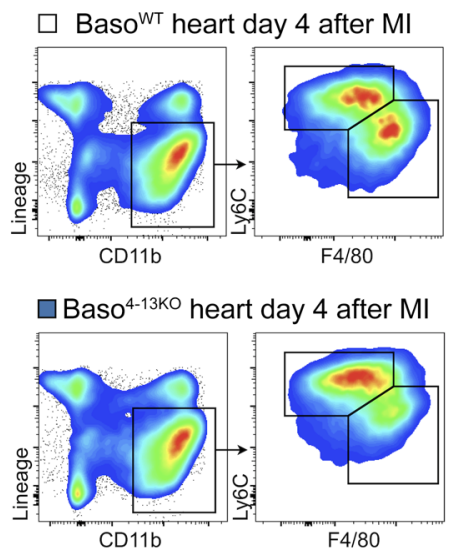

G

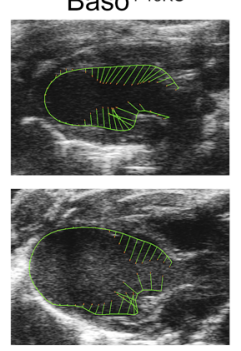

J

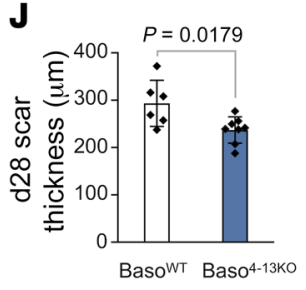

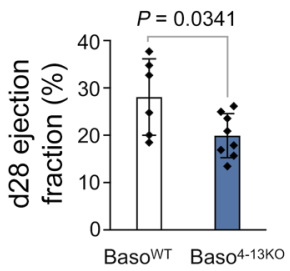

H

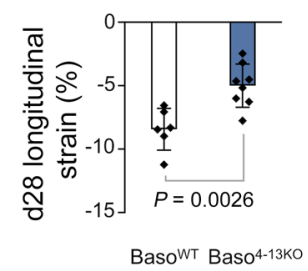

Figure 6. Basophil-specific IL-4/IL-13 depletion alters healing after MI. (A and B) Percentage of cardiac Ly6C $\mathrm{C}^{\text {hi }}$ monocytes and $\mathrm{Ly}_{6 \mathrm{C}}{ }^{\mathrm{lo}}$ macrophages (among the percentage of total Lin CD11b+ cells) 4 days after MI $(n=8-9)$ Data show the mean $\pm \mathrm{SD}$. $P$ values were determined by 2 -tailed Student's $t$ test. (C) Representative flow cytometric plots of cells from infarct tissue 4 days after $\mathrm{Ml}$ in Baso ${ }^{\mathrm{WT}}$ and Baso ${ }^{4-13 K 0}$ mice gated on monocytes/macrophages. (D and E) Echocardiographic evaluation of baseline EF and plasma cTnT levels 24 hours after LAD ligation in Baso ${ }^{W T}$ and Baso ${ }^{4-13 k 0}$ mice. (F) Representative echocardiographic images of mice from the indicated groups on day 1 and day 28 after MI. (G-I) Echocardiographic results for Baso ${ }^{W T}$ and Baso ${ }^{4-13 k 0}$ mice 4 weeks after $\mathrm{MI}(n=6-8)$. Data show the mean \pm SD. $P$ values were determined by 2-tailed Student's $t$ test. (J) Quantification of LV scar thickness based on histological evaluation 4 weeks after MI $(n=6-8)$. Data show the mean \pm SD. $P$ value was determined by 2 -tailed Student's $t$ test. (K) Representative images of histological sections stained with Masson's trichrome 4 weeks after MI. Scale bars: $500 \mu \mathrm{m}$.

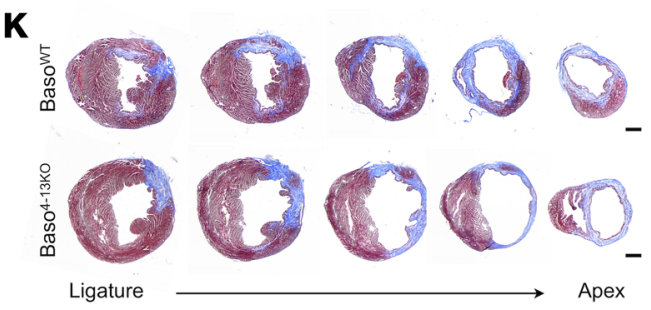

line characteristics are provided in Supplemental Table 3. Using linear regression analyses, we found that besides known risk factors, such as initial infarct size or inflammatory levels, low numbers of basophils in blood during the first week after MI correlated significantly with a larger scar size, quantified by late gadolinium enhancement (LGE) cardiac MRI after 1 year of follow-up $\left(R^{2}=\right.$ 0.1224, $P=0.0013$ ) (Figure 8, D-G, and Supplemental Table 4). Critically, this association persisted after adjustment of potential confounders, including initial infarct size, systemic inflammatory levels, and cardiovascular risk factors by multivariate regression analysis (Supplemental Table 5).

\section{Discussion}

Basophils are rare cells best known for their activity in allergic reactions and parasitic infections. Our knowledge about their function in the progression of cardiovascular diseases, however, is limited. Here, we report that basophils played an important role in regulating the post-MI immune response. Our in vivo studies indicated that the absence of basophils worsens the outcome after MI through an altered monocyte/macrophage response. Monocytes and monocyte-derived macrophages constitute the largest leukocyte subpopulations in the heart during the first days after cardiac ischemia and greatly affect adverse remodeling (27). Notably, we found that the absence of basophil accumulation substantially reduced the levels of IL-4/IL-13, which are prototypical type 2 cytokines and generally considered to have antiinflammatory properties. IL- 4 and IL-13 are known to induce phenotypical and functional changes within macrophages, which can then be characterized by high expression of classical antiinflammatory and tissue repair genes, such as Arg1, Mrc1 (CD206), or Retnla (28). Systemic IL-4 administration after MI was previously shown to reduce infarct size and enhance tissue repair, mainly through the augmentation of reparative macrophages $(29,30)$. IL-13 is also crucially involved in infarct healing, as genetic IL-13 deficiency worsens post-MI outcomes in adult mice (31).

Having shown that the absence of basophils significantly lowered IL-4/ IL-13 in the heart after MI, we next assessed the extent to which basophil-derived IL-4/IL-13 might be important in vivo. To do this, we generated mice with basophils lacking IL-4/IL-13. Basophil-specific IL-4/IL-13 deficiency recapitulated the altered monocyte/macrophage response and LV impairment previously observed in basophil-depleted mice. Yet, we cannot exclude the possibility that additional factors other than IL-4/IL-13 might contribute to the polarizing effects of basophils on macrophages. 


\section{A}
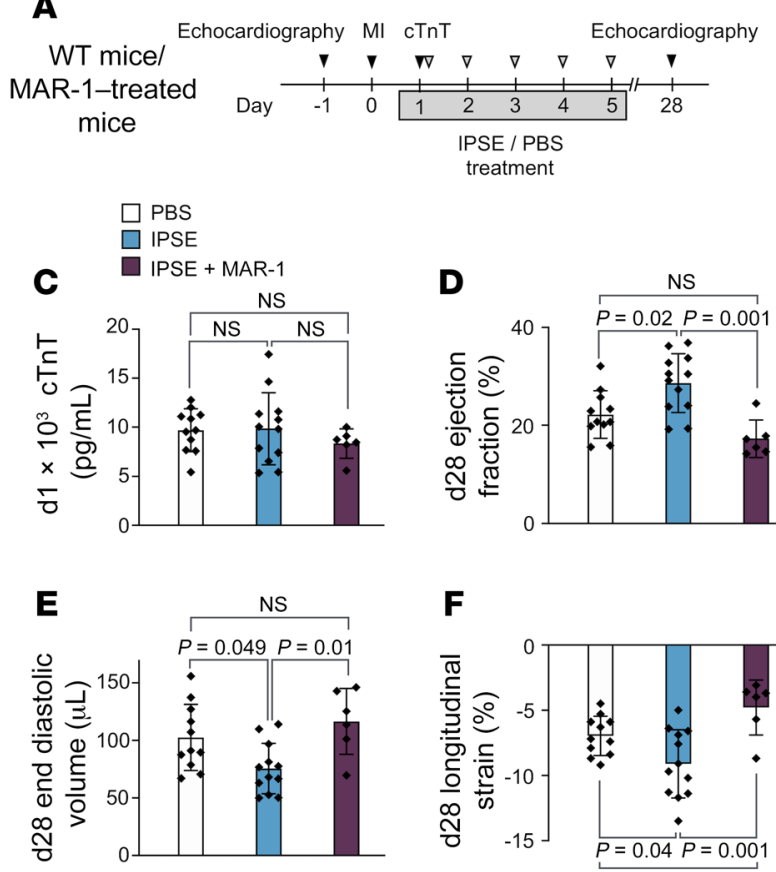

D
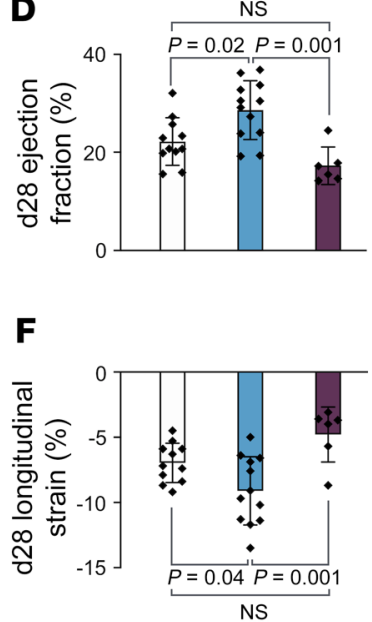

B
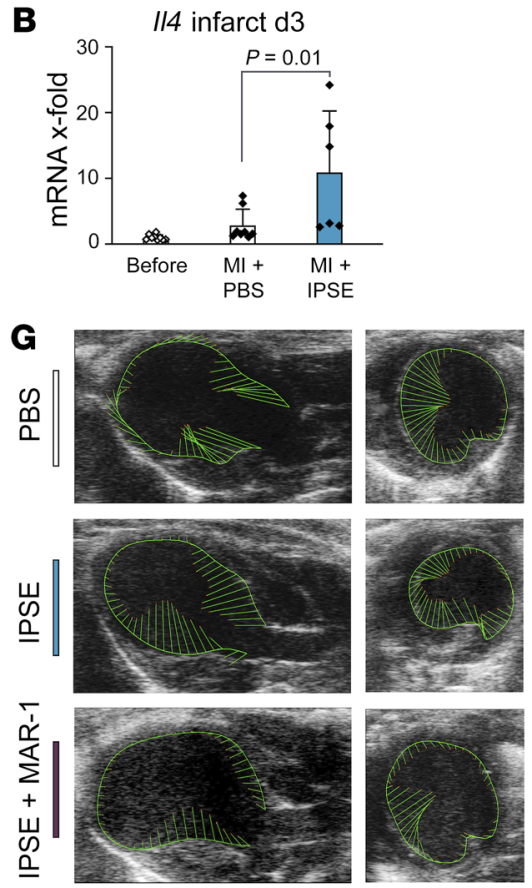

Figure 7. IPSE/ $\alpha-1$ enhances cardiac IL-4 and improves heart function after MI. (A) Timeline of experimental setting for the treatment of infarcted mice with IPSE/ $\alpha-1$ or PBS, respectively. (B) mRNA expression of $/ 14$ in the infarcted region of hearts from PBS- or IPSE/ $\alpha-1$-treated mice 3 days after MI. Data were pooled from 2 independent experiments $(n=6-8)$ and show the mean \pm SD. $P$ value was determined by 1-way ANOVA with Sidak's multiple-comparison test. (C) cTnT levels 1 day after LAD ligation. (D-F) Echocardiographic results for mice treated with either PBS, IPSE/ $\alpha-1$, or IPSE/ $\alpha-1$ plus MAR-1 twenty-eight days after MI $(n=6-12)$. Data show the mean \pm SD. $P$ values were determined by 1-way ANOVA with Tukey's multiple-comparison test. (G) Representative echocardiographic images of parasternal long-axis (left) and short-axis (right) views in mice from the indicated groups 4 weeks after experimental MI.

In addition, it is conceivable that basophil-derived IL-4 and IL-13 might also trigger the release of factors by macrophages themselves that promote their own phenotypic switch. Recently, it was shown that deficiency of IL-4 and IL-13 in hepcidin-deficient macrophages hampered tissue repair after $\mathrm{MI}$, suggesting that macrophages (especially CD206 ${ }^{+}$macrophages) are able to release IL-4 and IL-13 in the injured myocardium (32). Skewing themselves and neighboring macrophages toward a reparative phenotype via an autocrine/paracrine IL-4/IL-13 loop might be an additional mechanism for potentiating the effect of basophil-secreted IL-4 and IL-13. Finally, it should be noted that, apart from IL-4 and IL-13, other mechanisms have been described to be involved in macrophage polarization and fate specification after MI (33-36). It is tempting to speculate that some of these signals synergize with IL-4 and IL-13 to cooperatively induce antiinflammatory macrophage polarization. Interestingly, it has been shown that IL-4 and IL-13 require the presence of apoptotic and necroptotic cells to induce an antiinflammatory program in macrophages after microbial infection $(19,37)$. Further investigations will be required to fully decrypt how the different signals are integrated at cellular and molecular levels for post-MI macrophage reprogramming.

Parasites, and in particular schistosomes, have potent modulatory effects on the immune system by inducing robust type 2 immune responses. The major secreted product of schistosome eggs is the glycoprotein IPSE/ $\alpha-1$ (25). IPSE/ $\alpha-1$ activates basophils by antigen-independent binding to $\operatorname{IgE}$ molecules on the
FceRI and triggers the release of IL-4 and IL-13, thereby contributing to inflammation control in the host $(38,39)$. We evaluated whether these effects could also affect inflammation after cardiac ischemia and found beneficial effects upon administration of IPSE $/ \alpha-1$. Of note, the treatment was initiated after the infarct, which might suggest translational potential. Since basophil depletion reversed the improved outcome after the IPSE/ $\alpha-1$ regimen, we conclude that the protective effects were mediated primarily through the basophil IL-4/IL-13 axis. However, we cannot exclude the possibility that additional beneficial systemic effects might also have contributed to this protection, e.g., as it was recently shown that IPSE/ $\alpha-1$ can also trigger the development of regulatory B cells and their release of IL-10, or IPSE/ $\alpha-1$ might affect mast cells via FceRI signaling (40). Of note, we found that $\mathrm{IgE} / \mathrm{Fc}$ RI signaling was regulated after experimental $\mathrm{MI}$ in mice, even without IPSE/ $\alpha-1$ treatment, which was observed previously in patients with MI and might therefore be an interesting investigative target for future studies (41). Since these observations on upstream mechanisms for basophil activation and recruitment after MI are so far correlative, they will require further mechanistic validation in the future.

Our present study indicates that basophils have distinct kinetics in the blood in patients after ST-elevation MI (STEMI) and that an inadequate basophil response in humans correlates with impaired remodeling after acute MI. In contrast to our experiments in mice, however, no causative conclusions can be drawn regarding 
A

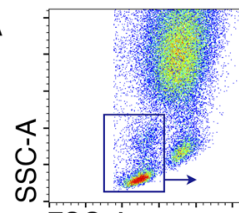

FSC-A

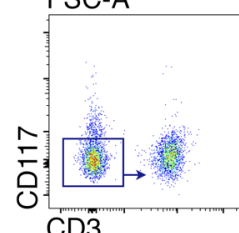

CD3

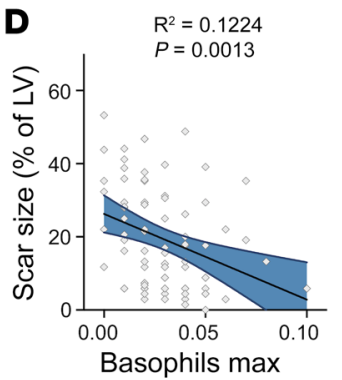

(cells/nL)
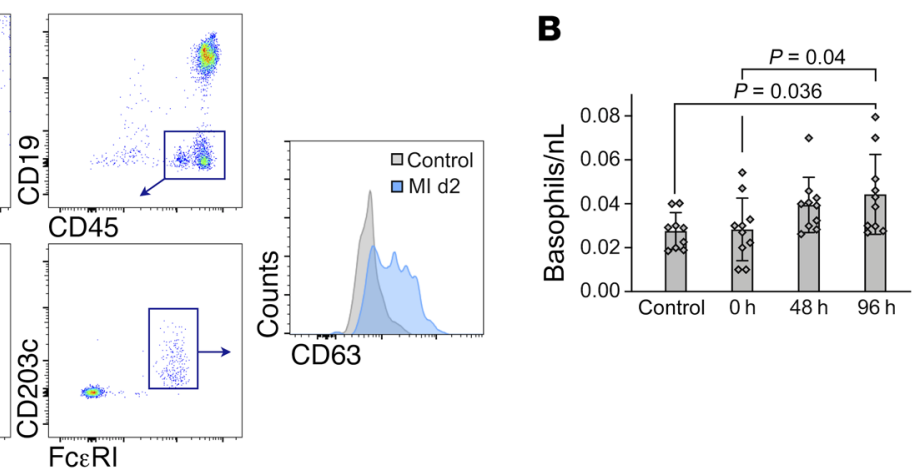

C
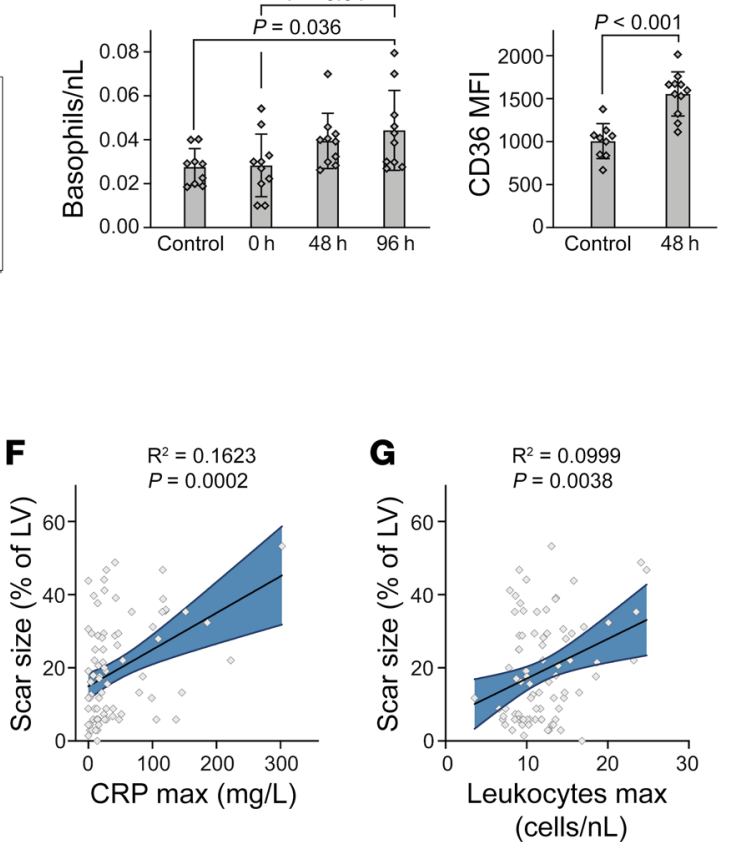

Figure 8. Low basophil counts predict poor remodeling in patients with STEMI. (A) Gating strategy for the identification of blood basophils in patients with STEMI. (B) Kinetics of blood basophils identified as CD45+CD19-CD3-CD117-FCERI+CD203c in patients with STEMI ( $n=11)$ and control patients $(n=9)$. $P$ values were determined by 1-way ANOVA with Sidak's multiple-comparison test. (C) Quantification of CD63 expression on human basophils based on MFI for control and MI patients. Data show the mean \pm SD. $P$ value was determined by 2-tailed Student's $t$ test. (D-C) Linear regression analysis for the association of scar size 12 months after MI and maximum (max) basophil counts during the first week after MI (D), cTnT levels 24 hours after MI (E), peak CRP values (F), and peak leukocyte counts (G) during the first week after Ml. Curved blue lines indicate $95 \%$ Cls. Each data point $(n=82)$ represents a biologically independent sample.

the association between basophil numbers and cardiac outcomes in humans. Further, we recognize that basophil kinetics including phenotypical changes in humans might be at least partially influenced by (so-far-unknown) effects of post-MI therapeutic regimens. It seems possible that IL-4/IL-13-driven pathologies such as atopic dermatitis and allergic asthma, but also their treatment, may interfere with the healing process after MI. This is of particular interest, as a growing number of patients will receive biological agents that interfere with the IL-4/IL-13 axis (e.g., the monoclonal antibody dupilumab). Further mechanistic studies are needed to identify possible interlinks and delineate the protective versus rather harmful effects of these conditions on post-MI healing.

In summary, we have identified an unexpected role for basophils in the context of MI healing. Despite their low numbers, we found that basophils strongly affected accumulating myeloid cells by releasing IL-4 and IL-13 and thereby influencing cardiac remodeling. More broadly, these findings provide insights into how basophils and basophil-derived cytokines control temporal and spatial containment of sterile inflammation. The use of basophils as regulators in Th1-driven pathologies might be a promising immune-modulatory therapeutic strategy for exaggerated acute or chronic inflammation.

\section{Methods}

Animals. All mice were on a C57BL/6J background and were studied at 8-10 weeks of age. Basophil-deficient Mcpt8-Cre (Baso-KO) mice
(14), IL-4eGFP reporter (4get) mice (42), and IL-4/IL-13-deficient mice (43) have been previously described. Animals were housed under standard laboratory conditions with a 12-hour light/12-hour dark cycle and ad libitum access to water and food.

Generation of mixed BM chimeras. BM cells were obtained from tibiae and femurs of Baso-KO mice and mixed at a 4:1 ratio with BM cells from either WT mice or IL-4/IL-13-deficient mice (24). Cells (2× $\left.10^{6}\right)$ were injected into the tail vein of lethally irradiated $(9 \mathrm{~Gy})$ recipient C57BL/6 mice to create Baso ${ }^{\mathrm{WT}}$ and Baso ${ }^{4-13 K o}$ mixed BM chimeras (MBMCs), respectively. Mice were given antibiotic-containing drinking water $(2 \mathrm{~g} / \mathrm{L}$ neomycin sulfate, $100 \mathrm{mg} / \mathrm{L}$ polymyxin B 328 sulfate; MilliporeSigma) for 8 weeks after reconstitution.

Induction of MI. MI was induced by permanent ligation of the LAD as described previously (35). In brief, anesthesia was induced with isoflurane $\left(4 \% / 800 \mathrm{~mL} \mathrm{O}_{2} / \mathrm{min}\right)$ and maintained by endotracheal ventilation $\left(2 \%-3 \% / 800 \mathrm{~mL} \mathrm{O}_{2} / \mathrm{min}\right)$. A thoracotomy was performed in the fourth left intercostal space. The left ventricle was exposed, and the left coronary artery was permanently occluded. The chest and skin were closed, and anesthesia was terminated. The animals were extubated when their breathing was restored. Initial myocardial injury was evaluated by measuring cTnT levels in plasma 24 hours after MI induction. Valid myocardial injury was defined as a cTnT level above $5000 \mathrm{pg} / \mathrm{mL}$. Sham-operated animals underwent the same procedure except for the LAD occlusion.

Basophil depletion and basophil-specific IL-4 induction. For depletion of basophils, $10 \mu \mathrm{g}$ anti-Fc\&RI antibody (eBiosciences, clone 
MAR-1) was injected i.p. on pre-MI days $-3,-2$, and -1 and repeated on post-MI days 1 and 5 in order to maintain reduced levels during the first 7 days after MI. IPSE/ $\alpha-1$ was expressed in human embryonic kidney (HEK) cells and purified from culture supernatant as described previously (44). Mice were then injected i.v. with $25 \mu \mathrm{g}$ IPSE $/ \alpha-1$ or saline on post-MI days 1, 2, 3, 4, and 5 .

Adoptive transfer of basophil-enriched cells. Adoptive transfer of basophil-enriched cells was performed by combining 2 previously published methods $(15,20,45)$. In detail, donor WT mice were injected i.v. with a combination of $10 \mu \mathrm{g}$ mouse recombinant IL-3 (Peprotech) and $5 \mu \mathrm{g}$ anti-IL-3 antibody (BD Biosciences, clone MP2-8F8) in 200 $\mu \mathrm{L}$ PBS 3 days before euthanasia in order to increase the basophil fraction within these mice. At necropsy, single-cell suspensions of spleens were prepared, and the basophil-enriched $\mathrm{CD} 49 \mathrm{~b}^{+}$fraction was isolated using PE-conjugated anti-NK-1.1 antibodies (BD Biosciences, clone PK136) and anti-phycoerythrin (anti-PE) MicroBeads (Miltenyi Biotec) for negative selection and anti-CD49b MicroBeads (Miltenyi Biotec, clone DX5) for positive selection. Cells $\left(1.0 \times 10^{6}\right)$ were resuspended in $200 \mu \mathrm{L}$ DMEM and transferred into Baso-KO mice i.v. on days 1, 2, and 3 after experimental MI.

Echocardiographic measurements. Echocardiography was performed on a Vevo 2100 (VisualSonics). Mice were conscious during the echocardiographic measurements. Left parasternal long-axis and left mid-papillary views were acquired. LV end-diastolic volume, end-systolic volume, and EF were evaluated on the left parasternal long-axis view using VevoLab software (VisualSonics). Longitudinal LV global strain was quantified in the longitudinal axis by speckle tracking using VevoStrain software (VisualSonics). Infarct expansion was quantified semiautomatically by analyzing regional displacement in the parasternal long axis using VevoStrain software. The infarct region was defined as the percentage of $\mathrm{LV}$ area with a displacement of less than 0.1 during mid-systole relative to the global LV circumference (49 LV spots). Infarct expansion was defined as $\Delta=$ infarct region (day 28) - infarct region (day 1). Investigators were blinded to the sample group allocation during the experiments and analyses.

Histopathological analysis. Post-MI cardiac healing was assessed on day 28. For paraffinized sections, hearts were excised, rinsed in PBS, and fixed for 1-3 days in $10 \%$ buffered formalin at $4^{\circ} \mathrm{C}$. Hearts were then dehydrated, paraffinized, and sectioned (4-6 $\mu \mathrm{m})$. For cryosections, hearts were excised, rinsed in PBS, fixed for 3 hours in $4 \%$ PFA at room temperature, and stored overnight in $30 \%$ sucrose at $4^{\circ} \mathrm{C}$. Hearts were embedded in Tissue-Tek OCT compound (Sakura) and sectioned ( $9 \mu \mathrm{m}$ thickness). Tissue sections were then stained with the Masson's Trichrome Stain Kit (MilliporeSigma) according to the manufacturer's instructions. Image J software (NIH) was used to analyze the fibrotic area. Scar thickness was averaged from 5 measurements at $1 \mathrm{~mm}$ intervals from the ligature site to the apex in both long and short axes in a blinded fashion.

Multiplex immunohistochemical analysis. Multiplex immunohistochemical stainings of $4 \mu \mathrm{m}$ sections from murine infarcted hearts or healthy specimens were performed using the BOND RX Research Stainer (Leica Biosystems) with antibodies against Mcpt8 (BioLegend, clone TUG8, 1:100 dilution), IL-4 (BioLegend, clone 11B11, 1:250 dilution), CD163 (Abcam, clone EPR19518, 1:100 dilution), and IgE (BioLegend, clone RME-1, 1:250 dilution) according to the manufacturer's instructions. Recommended solutions from the Opal Polaris Color Automation IHC Detection Kit (Akoya Biosciences) as well as
Opal fluorophores from the Opal 7-Color Fluorescent IHC Kit (PerkinElmer) were used. DAPI was used for nuclear visualization, and slides were mounted with Fluoromount-G (SouthernBiotech). Images were acquired using the Vectra Polaris automated imaging system at $\times 20$ resolution. Images were analyzed using Inform software, version 2.4 (Akoya Biosciences) and quantified using QuPath (46).

Flow cytometry. Peripheral blood was collected by facial vein puncture in heparinized tubes, and erythrocytes were lysed in RBC lysis buffer (Miltenyi Biotec). Hearts, spleens, and BM were harvested after cervical dislocation. Single-cell suspensions of $\mathrm{BM}$ were obtained by flushing 1 dissected femur with $2 \mathrm{~mL}$ ice-cold PBS (MilliporeSigma). Single-cell suspensions of infarcted hearts were obtained by mincing the tissue with fine scissors and digesting it with a solution containing $450 \mathrm{U} / \mathrm{mL}$ collagenase I, $125 \mathrm{U} / \mathrm{mL}$ collagenase $\mathrm{XI}, 60 \mathrm{U} / \mathrm{mL}$ DNase I, and $60 \mathrm{U} / \mathrm{mL}$ hyaluronidase (MilliporeSigma) for 1 hour at $37^{\circ} \mathrm{C}$ while shaking. Absolute cell numbers were counted in a Neubauer chamber. Cells $\left(10 \times 10^{6}\right)$ were stained for flow cytometric analysis. The fluorescent antibodies and corresponding gating strategies used are described in the Supplemental Methods. Flow cytometry was performed on a FACSVerse (BD Biosciences). Data were analyzed using FlowJo software.

Quantitative real-time PCR. Total RNA was isolated from cardiac tissue using TRIzol Reagent (Thermo Fischer Scientific) following the manufacturer's instructions. RNA was reverse transcribed using the Revert Aid First Strand cDNA Synthesis Kit (Thermo Fisher Scientific). Quantitative real-time PCR (RT-qPCR) was performed using SYBR Green (Bio-Rad Laboratories) according to the manufacturer's instructions. Relative changes were normalized to $18 \mathrm{~s}$ mRNA using the $2^{\Delta \Delta \mathrm{Ct}}$ method. mRNA levels are expressed relative to the indicated control conditions (set to 1). All primers are listed in the Supplemental Methods.

In silico analysis of single-cell RNA-Seq. The ArrayExpress data set E-MTAB-7376 was examined. This data set contains sequencing data from nonmyocytic cells isolated from hearts on days 3 and 7 after permanent ligation of the LAD (21). Analysis was performed using the Python package Scanpy (47). Only cells with more than 200 expressed genes and a mitochondrial content of less than $10 \%$ were considered. Genes that were expressed in fewer than 3 cells were also filtered out. The data were log-normalized to a scale factor of 10,000. Only highly variable genes were considered for linear dimensional reduction and were defined by a minimum mean expression of 0.0125, a maximum mean expression of 3 , and a minimum dispersion of 0.5 . The effects of total counts and mitochondrial content were regressed out. The dimensionality reduction was done using principal component analysis (PCA). The neighborhood graph was computed using the UMAP algorithm for the first 50 principal components $\left(n_{-} P C=50\right)$. Unsupervised clustering was performed using the Leiden algorithm. Differentially expressed genes were calculated for each cluster using the Wilcoxon rank-sum test. Clusters enriched for leukocyte markers were selected. This leukocyte subpopulation was then reanalyzed for day 7 (n_PC = 20) and a combination of days 3 and day 7 (for evaluation of cytokine/cytokine receptor expression; n_PC $=40$ ) for different cell types as described above, and each cluster was annotated to a specific cell type.

Population of patients with acute MI. Blood samples from 11 consecutive patients with acute STEMI and 9 matched control patients with nonischemic cardiovascular disease were analyzed by flow cytometry. All patients with MI had been treated by percutaneous 
coronary intervention (PCI) and stenting within a median of 3 hours of symptom onset. Heparinized blood was lysed using BD FACS lysing solution (BD Biosciences) according to the manufacturer's instructions. The following fluorescent antibodies were used for staining and flow cytometric analysis: anti-CD45-FITC (BioLegend, clone HI30), anti-CD19-APC-Cy7 (BioLegend, clone HIB19), antiCD3-Percp-Cy5.5 (BioLegend, clone UCHT1), anti-CD117-PE-Cy7 (BioLegend, clone 104D2), anti-FceRI-PE (BioLegend, clone AER37), anti-CD203c-APC (BioLegend, clone NP4D6), and anti-CD63BV421 (BioLegend, clone H5C6). Human basophils were identified as $\mathrm{CD}^{-} 5^{+} \mathrm{CD} 19^{-} \mathrm{CD}^{-} \mathrm{CD} 117^{-} \mathrm{Fc} \mathrm{RII}^{+} \mathrm{CD} 2 \mathrm{O} 3 \mathrm{c}^{+}$cells (48). Flow cytometry was performed on a FACSVerse flow cytometer (BD Biosciences). Data were analyzed using FlowJo software. Another 82 patients with acute MI were separately considered in our observational follow-up study. Patients who presented with acute STEMI at the chest pain unit of the University Hospital of Heidelberg were included from November 2010 until January 2020. All patients underwent PCI and stenting and were treated according to European Society of Cardiology (ESC) guidelines, which included administration of aspirin, P2Y12 antagonists, heparin, morphine, beta blockers, statins, and - if the EF was lower than 55\% - angiotensin-converting enzyme (ACE) inhibitors or AT1 antagonists, as well as mineralocorticoid antagonists for heart failure therapy. Patients undergoing immunosuppressive therapy were excluded from the analysis. Basophils were counted on an ADVIA 2120i hematology system (Siemens) using the basophil/lobularity method, which allows valid identification of basophils in human blood. Heart characteristics were determined by cardiac magnetic resonance (CMR) 12 months after the initial event. The association of each covariate with the dependent variable was assessed with a univariate linear regression model. Bonferroni's correction was used to adjust the $P$ values for multiple testing. The covariates are listed in Supplemental Table 4 and included maximum basophil counts, cTnT levels 24 hours after MI, as well as maximum peripheral leukocyte counts and serum C-reactive protein (CRP) values during the first week after MI. Multivariate regression analysis was performed after computed variable selection. In detail, all variables with a $P$ value of greater than 0.5 in the univariate linear regressions were excluded. Then, backward selection was performed, and the variable with the highest $P$ value was excluded. The backward steps were repeated until all included variables were significant $(P<0.05)$.

Statistics. Statistical analyses were performed using GraphPad Prism, version 8 (GraphPad Software). Endpoint comparisons between
2 groups were performed using an unpaired, 2-tailed Student's $t$ test after passing normal distribution testing. Differences between more than 2 groups were analyzed by 1-way or 2-way ANOVA followed by Tukey's or Sidak's post hoc test. A P of less than 0.05 was considered statistically significant. All data are expressed as the mean \pm SD.

Study approval. All animal procedures were approved by the IRB of the University of Heidelberg and the responsible government authority (Regierungspräsidium Karlsruhe of the state of Baden-Württemberg, Karlsruhe, Germany; project nos. G-318/14 and G-43/17). Human studies were conducted according to Declaration of Helsinki principles and were approved by the local ethics commission (Ethikkommission der Medizinischen Fakultät Heidelberg, Heidelberg, Germany). Written informed consent was obtained from all patients.

\section{Author contributions}

FS, ISM, NF, HAK, DV, and FL conceived and designed the study. ISM, XL, and YZ performed LAD ligation surgery. FS, MK, TCK, and FL performed the echocardiographic studies and analyzed the results. FS, ISM, XL, CM, JKM, JP, and FL the performed flow cytometric and immunohistochemical experiments. FS and XL performed histopathological evaluations. DR and DV generated the MBMCs. SD, KJL, TT, and MB analyzed the single-cell RNA-Seq data sets and human data. GS provided IPSE/ $\alpha-1$ and performed in vitro experiments with human basophils. All authors contributed to the preparation of the manuscript.

\section{Acknowledgments}

The authors thank Susann Werkmeister for her excellent technical assistance. This work was supported by the German Research Foundation (DFG) (Heisenberg Programme and LE 2530/5-1, to FL; SFB 850 and SFB160, to MB); the Leducq Foundation (The Inflammatory-Fibrosis Axis in Ischemic Heart Failure, to KJL and FL); the German Cardiac Society (DGK) (Otto-Hess scholarship, to FS); and the German Federal Ministry of Education and Research within the Medical Informatics Funding Scheme (MIRACUM) (FKZ 01ZZ1606B, to MB); and was conducted within the framework of CoNfirm (FKZ 01ZX1708F, to FL, MB, and SD).

Address correspondence to: Florian Leuschner, Department of Cardiology, University Hospital Heidelberg, Im Neuenheimer Feld 410, 69120 Heidelberg, Germany. Phone: 0049.6221.56.38869; Email: florian.leuschner@med.uni-heidelberg.de.
1. Frangogiannis NG. The inflammatory response in myocardial injury, repair, and remodelling. Nat Rev Cardiol. 2014;11(5):255-265.

2. Horckmans M, et al. Neutrophils orchestrate post-myocardial infarction healing by polarizing macrophages towards a reparative phenotype. Eur Heart J. 2017;38(3):187-197.

3. Bajpai G, et al. Tissue resident CCR2 ${ }^{-}$and $\mathrm{CCR} 2^{+}$cardiac macrophages differentially orchestrate monocyte recruitment and fate specification following myocardial injury. Circ Res. 2019;124(2):263-278.

4. Hilgendorf I, et al. Ly-6Chigh monocytes depend on Nr4a1 to balance both inflammatory and reparative phases in the infarcted myocardium. Circ Res. 2014;114(10):1611-1622.
5. Libby P, et al. Leukocytes link local and systemic inflammation in ischemic cardiovascular disease: an expanded "cardiovascular continuum." JAm Coll Cardiol. 2016;67(9):1091-1103.

6. Hofmann U, et al. Activation of $\mathrm{CD}^{+}{ }^{+} \mathrm{T}$ lymphocytes improves wound healing and survival after experimental myocardial infarction in mice. Circulation. 2012;125(13):1652-1663.

7. Weirather J, et al. Foxp $3^{+} \mathrm{CD} 4^{+} \mathrm{T}$ cells improve healing after myocardial infarction by modulating monocyte/macrophage differentiation. Circ Res. 2014;115(1):55-67.

8. Zouggari Y, et al. B lymphocytes trigger monocyte mobilization and impair heart function after acute myocardial infarction. Nat Med. 2013;19(10):1273-1280.
9. Ngkelo A, et al. Mast cells regulate myofilament calcium sensitization and heart function after myocardial infarction. J Exp Med. 2016;213(7):1353-1374.

10. Anzai A, et al. Regulatory role of dendritic cells in postinfarction healing and left ventricular remodeling. Circulation. 2012;125(10):1234-1245.

11. Ehrlich P. Ueber die specifischen Granulationen des Blutes. Archiv fuer Anatomie und Physiologie: Physiologische Abteilung. 1879;571-579.

12. Sullivan BM, Locksley RM. Basophils: a nonredundant contributor to host immunity. Immunity. 2009;30(1):12-20.

13. Voehringer D. Protective and pathological roles of mast cells and basophils. Nat Rev Immunol. 2013;13(5):362-375. 
14. Ohnmacht C, et al. Basophils orchestrate chronic allergic dermatitis and protective immunity against helminths. Immunity. 2010;33(3):364-374

15. Wada $\mathrm{T}$, et al. Selective ablation of basophils in mice reveals their nonredundant role in acquired immunity against ticks. JClin Invest. 2010;120(8):2867-2875.

16. Charles N, et al. Basophils and the Thelper 2 environment can promote the development of lupus nephritis. Nat Med.2010;16(6):701-707.

17. Cohen $\mathrm{M}$, et al. Lung single-cell signaling interaction map reveals basophil role in macrophage imprinting. Cell. 2018;175(4):1031-1044.

18. Schiechl G, et al. Basophils trigger fibroblast activation in cardiac allograft fibrosis development. Am J Transplant. 2016;16(9):2574-2588.

19. Bleriot $\mathrm{C}$, et al. Liver-resident macrophage necroptosis orchestrates type 1 microbicidal inflammation and type-2-mediated tissue repair during bacterial infection. Immunity. 2015;42(1):145-158.

20. Piliponsky AM, et al. Basophil-derived tumor necrosis factor can enhance survival in a sepsis model in mice. Nat Immunol. 2019;20(2):129-140.

21. Farbehi N, et al. Single-cell expression profiling reveals dynamic flux of cardiac stromal, vascular and immune cells in health and injury. Elife. 2019;8:e43882.

22. Voehringer D. Basophil modulation by cytokine instruction. Eur JImmunol. 2012;42(10):2544-2550.

23. Dunkelberger JR, Song WC. Complement and its role in innate and adaptive immune responses. Cell Res. 2010;20(1):34-50.

24. Schwartz C, et al. Basophil-mediated protection against gastrointestinal helminths requires IgE-induced cytokine secretion. Proc Natl Acad Sci U S A. 2014;111(48):E5169-E5177.

25. Schramm G, et al. IPSE/alpha-1: a major immunogenic component secreted from Schistosoma mansoni eggs. Mol Biochem Parasitol. 2006;147(1):9-19.

26. Schramm G, et al. Molecular characterization of an interleukin-4-inducing factor from Schistosoma mansoni eggs. J Biol Chem. 2003;278(20):18384-18392.
27. Leuschner F, Nahrendorf M. Novel functions of macrophages in the heart: insights into electrical conduction, stress, and diastolic dysfunction. Eur Heart J. 2020;41(9):989-994.

28. Van Dyken SJ, Locksley RM. Interleukin-4- and interleukin-13-mediated alternatively activated macrophages: roles in homeostasis and disease. Annu Rev Immunol. 2013;31:317-343.

29. Shiraishi M, et al. Alternatively activated macrophages determine repair of the infarcted adult murine heart. JClin Invest. 2016;126(6):2151-2166

30. Shintani Y, et al. IL-4 as a repurposed biological drug for myocardial infarction through augmentation of reparative cardiac macrophages: proofof-concept data in mice. Sci Rep. 2017;7(1):6877.

31. Hofmann $\mathrm{U}$, et al. Interleukin-13 deficiency aggravates healing and remodeling in male mice after experimental myocardial infarction. Circ Heart Fail. 2014;7(5):822-830.

32. Zlatanova I, et al. Iron regulator hepcidin impairs macrophage-dependent cardiac repair after injury. Circulation. 2019;139(12):1530-1547.

33. Zhang S, et al. Efferocytosis fuels requirements of fatty acid oxidation and the electron transport chain to polarize macrophages for tissue repair. Cell Metab. 2019;29(2):443-456.

34. Shirakawa K, et al. IL (Interleukin)-10-STAT3galectin-3 axis is essential for osteopontinproducing reparative macrophage polarization after myocardial infarction. Circulation. 2018;138(18):2021-2035.

35. Meyer IS, et al. The cardiac microenvironment uses non-canonical WNT signaling to activate monocytes after myocardial infarction. EMBO Mol Med. 2017;9(9):1279-1293.

36. Ma Y, et al. Matrix metalloproteinase- 28 deletion exacerbates cardiac dysfunction and rupture after myocardial infarction in mice by inhibiting M2 macrophage activation. Circ Res. 2013;112(4):675-688.

37. Bosurgi L, et al. Macrophage function in tissue repair and remodeling requires IL-4 or IL-13 with apoptotic cells. Science. 2017;356(6342):1072-1076.
38. Knuhr K, et al. Schistosoma mansoni egg-released IPSE/alpha-1 dampens inflammatory cytokine responses via basophil interleukin (IL)-4 and IL-13. Front Immunol. 2018;9:2293.

39. Meyer NH, et al. A crystallin fold in the interleukin-4-inducing principle of Schistosoma mansoni eggs (IPSE/ $\alpha-1$ ) mediates IgE binding for antigen-independent basophil activation. J Biol Chem. 2015;290(36):22111-22126.

40. Haeberlein S, et al. Schistosome egg antigens, including the glycoprotein IPSE/alpha-1, trigger the development of regulatory B cells. PLOS Pathog. 2017;13(7):e1006539.

41. Szczeklik A, et al. Serum immunoglobulin E response to myocardial infarction. Circulation. 1988;77(6):1245-1249.

42. Mohrs M, et al. Analysis of type 2 immunity in vivo with a bicistronic IL-4 reporter. Immunity. 2001;15(2):303-311.

43. McKenzie GJ, et al. Simultaneous disruption of interleukin (IL)-4 and IL-13 defines individual roles in Thelper cell type 2-mediated responses. JExp Med.1999;189(10):1565-1572.

44. Schramm G, et al. Cutting edge: IPSE/alpha-1, a glycoprotein from Schistosoma mansoni eggs, induces IgE-dependent, antigen-independent IL-4 production by murine basophils in vivo. JImmunol. 2007;178(10):6023-6027.

45. Ohmori K, et al. IL-3 induces basophil expansion in vivo by directing granulocyte-monocyte progenitors to differentiate into basophil lineage-restricted progenitors in the bone marrow and by increasing the number of basophil/ mast cell progenitors in the spleen. JImmunol. 2009;182(5):2835-2841.

46. Bankhead P, et al. QuPath: open source software for digital pathology image analysis. Sci Rep. 2017;7(1):16878.

47. Wolf FA, et al. SCANPY: large-scale single-cell gene expression data analysis. Genome Biol. 2018;19(1):15.

48. Sharma M, et al. Regulatory T cells induce activation rather than suppression of human basophils. Sci Immunol. 2018;3(23):eaan0829. 